\title{
TUMORES DE CÉlULAS GERMINATIVAS PRIMÁRIOS DO MEDIASTINO
}

\author{
Wilson José Couto*, Jefferson luiz Gross, Daniel Deheinzelin, Riad Naim Younes
}

Trabalho realizado no departamento de Cirurgia Torácica do Centro de Tratamento e Pesquisa do Hospital do Câncer A.C. Camargo, São Paulo, SP

\author{
*Correspondência: \\ Hospital do Câncer A.C. \\ Camargo \\ Departamento de Cirurgia \\ Torácica \\ Rua Prof. Antonio Prudente, \\ 211, Liberdade \\ Tel: (11) 3272-5119 \\ Fax: (11) 3272-5135 \\ Cep: 01509-900, \\ São Paulo, SP
}

\begin{abstract}
RESUMO
Os tumores de células germinativas primários do mediastino podem corresponder de 10\% a 20\% das neoplasias mediastinais. Ocorrem devido à transformação maligna de elementos germinativos sem evidência de um foco gonadal primário. Podem ser classificados de acordo com suas características morfológicas em: teratomas, seminomas, não seminomas (tumores do saco vitelino, carcinomas embrionários e coriocarcicoma), e tumores de células germinativas combinados sem componentes teratomatosos. O objetivo dessa revisão é analisar os diferentes tipos de tumores de células germinativas primários do mediastino no que diz respeito aos seus aspectos clínicos bem como as suas diferentes formas de abordagens terapêuticas.
\end{abstract}

Unitermos: Tumores de células germinativas. Mediastino. Classificação. Teratomas. Seminomas. Não seminomas.

\section{INTRODUÇÃo}

Os tumores de células germinativas (TCG) são neoplasias pouco comuns que acometem mais freqüentemente as gônadas, embora possam também ocorrer em outras localizações, como a glândula pineal, o retroperitôneo, o mediastino e a área sacral'.

Os tumores de células germinativas primários do mediastino (TCGM) ocorrem principalmente no mediastino anterior e podem corresponder de 10\% a 20\% de todas as neoplasias mediastinais!.

Inicialmente, acreditava-se que a ocorrência dos TCG no mediastino se devesse à disseminação de um tumor germinativo gonadal primário, considerando a semelhança histopatológica entre esses tumores!.

Mais recentemente, acredita-se que os TCG primários extragonodais derivam de células germinativas primitivas que migraram ao longo da crista urogenital durante a embriogênese precoce ou distribuíram-se fisiologicamente para o figado, medula espinhal e cérebro, a fim de desempenhar funções regulares ou transportar informações hematológicas ou imunológicas. A anomalia genética característica observada nos TCG, tanto gonadais como extragonodais, é um isocromossomo i $(12 p)^{3}$. Portanto, acredita-se atualmente que os tumores de células germinativas extragonodais ocorram devido à transformação maligna de elementos germinativos sem a evidência de um foco gonadal. Como confirmação de tal teoria, cita-se a presença da associação de TCGM não seminomatosos com a síndrome de Klinefelter e também a associação exclusiva desses tumores com neoplasias hematológicas².

Em geral, os TCGM podem ser classificados em seminomatosos e não seminomatosos, e são muito semelhantes histologicamente aos TCG gonadais².

Moran' divide os tumores de células germinativas do mediastino em duas categorias principais, conforme as suas características histopatológicas: I) lesões teratomatosas e 2) lesões não

\section{Tabela I - Classificação histopatológica dos tumores de células germinativas do mediastino}

\section{Tumores teratomatosos}

Teratomas maduros: compostos de elementos maduros, bem diferenciados

Teratomas imaturos: com a presença de tecidos mesenquimal ou neuropitelial imaturos

\section{Teratomas com componentes malignos adicionais}

Tipo I: com outro tumor de células germinativas (seminoma, carcinoma embrionário, tumor de saco vitelino, etc)

Tipo II: com componente epitelial não germinativo (carcinoma epidermóide, adenocarcinoma, etc)

Tipo Ill: com componente maligno mesenquimal(rabdomiossarcoma, condrossarcoma, etc) Tipo IV: com qualquer combinação dos citados acima

\section{Tumores não teratomatosos}

Seminomas

Tumores de saco vitelino

Carcinomas embrionários

Coriocarcinomas

Tumores não teratomatosos combinados (qualquer combinação dos citados acima)

Fonte: Moran CA, Suster S. Primary germ cell tumors of the mediastinum I - Analysis of 322 cases with special emphasis on teratomatous lesions and proposal for histopathologic classification and clinical staging. Cancer 1997; 80:681-90

teratomatosas, que incluem: seminomas, tumores de células germinativas não seminomatosos e não teratomatosos (tumores do saco vitelino, carcinomas embrionários e coriocarcinomas), e os tumores de células germinativas combinados sem componentes teratomatosos (Tabela I).

Os teratomas com componentes malignos foram subclassificados de acordo com os elementos presentes: epitelial, mesenquimal, outro componente maligno de células germinativas ou a combinação desses tipos (Tabela I). 
O mesmo autor' propôs um esquema de estadiamento clínico desses tumores com a divisão nos seguintes grupos: estadio I para os tumores restritos ao mediastino sem envolvimento de estruturas adjacentes; estadio II para aqueles restritos ao mediastino com evidência macroscópica ou microscópica de infiltração em estruturas vizinhas como pleura, pericárdio, grandes vasos, etc; e estadio III para os tumores com evidência de metástases intra ou extratorácicas (Tabela 2).

Faremos uma descrição dos principais tipos de TCGM com base em suas características clínicas.

\section{Teratomas}

O teratoma cístico maduro é o tipo mais comum de TCGM. Normalmente ocorre em adultos jovens e a maioria encontra-se no

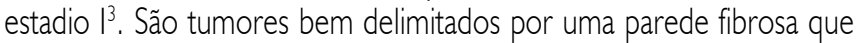
freqüentemente adere às estruturas ao redor e pode tornar-se calcificada. A rotura do conteúdo cístico na árvore traqueobrônquica, secundária à erosão, pode levar à tosse com eliminação de conteúdo sebáceo oleoso e até cabelos ${ }^{3}$.

Os teratomas imaturos e os teratocarcinomas afetam preferencialmente homens jovens ( 15 a 35 anos) e ocasionalmente mulheres. Caracterizam-se por serem massas multilobulares, algumas vezes bem encapsuladas e freqüentemente císticas. Geralmente apresentam metástases hematógenas precocemente e apresentam a alfafetoproteína (AFP), a gonadotrofina coriônica humana beta (b-HCG) e o antígeno carcinoembrionário (CEA) ocasionalmente elevados².

Nos teratomas imaturos puros, o prognóstico freqüentemente é bom, dependendo em geral da idade do paciente (prognóstico melhor abaixo dos 15 anos de idade)2.

Os teratomas com componentes malignos podem ser subdivididos também com base nos tipos de neoplasia associada aos componentes benignos. Podem também apresentar evidência macroscópica de invasão de estruturas adjacentes incluindo-se a pleura, pulmão e osso'.

\section{Seminomas}

A presença de seminoma primário de mediastino foi descrita por Woolmer et al. em 19554.

Os seminomas ocorrem preferencialmente em homens jovens ( 15 a 35 anos de idade) e raramente em mulheres, quando são conhecidos como disgerminomas ${ }^{2}$.

Estudos moleculares indicam que os seminomas mediastinais representam uma entidade distinta e separada dos seminomas gonadais. Os seminomas são os segundos TCGM em sua forma pura, seguindo-se aos teratomas ${ }^{5}$.

Clinicamente os seminomas mediastinais caracterizam-se pela presença de massas mediastinais de localização anterior. A presença de sintomas está relacionada com o tamanho do tumor, sendo a maioria dos sintomas, como tosse, dor torácica, hemoptise ou dispnéia, secundária à compressão de estruturas adjacentes. Em alguns casos, o quadro clínico pode ser agudo como a presença de síndrome de veia cava superior. Em outros, está associado com características clínicas pouco comuns como a história de trauma testicular, orquite ou de testículos com descida incompleta. Várias síndromes clínicas também podem estar associadas com os seminomas mediastinais,

\section{Tabela 2 - Estadiamento clínico dos tumores de células germinativas do mediastino}

Estadio I: Tumores bem circunscritos com ou sem adesões focais para a pleura ou pericárdio, mas sem evidência microscópica de invasão em estruturas adjacentes.

Estadio Il: Tumor restrito ao mediastino com evidência de infiltração macroscópica e/ou microscópica em estruturas adjacentes (como a pleura, pericárdio e grandes vasos)

Estadio Ill: Tumor com metástases:

A - para órgãos intratóracicos (linfonodos, pulmão, etc)

B - extratorácicas

Fonte: Moran CA, Suster S. Primary germ cell tumors of the mediastinum I - Analysis of 322 cases with special emphasis on teratomatous lesions and proposal for histopathologic classification and clinical staging. Cancer 1997; 80:681-90

como comunicação interventricular, ausência congênita de hemi-vértebra torácica, estenose pulmonar e ginecomastia ${ }^{5}$.

Os seminomas mediastinais também podem ser completamente assintomáticos e achados acidentalmente em exames rotineiros. Radiologicamente esses tumores apresentam-se como massas grandes, bem circunscritas, que se estendem a ambos os lados da linha média. Na tomografia axial computadorizada de tórax, os seminomas são grandes, coalescentes, lobulados e com atenuação homogênea semelhante à de partes moles. A B-HCG sérica pode ocasionalmente estar elevada².

O diagnóstico diferencial dos seminomas mediastinais inclui uma variedade de neoplasias primárias e metastáticas incluindo-se timomas, carcinomas tímicos, linfomas e melanomas. Entretanto, a possibilidade de metástase de um tumor primário testicular, embora rara, sempre deve ser considerada. Portanto, são importantes uma avaliação clínica cuidadosa e exame físico minucioso desses pacientes 5 .

Os estadiamentos clínico e patológico são os indicadores prognósticos mais importantes nos pacientes com seminomas mediastinais. Aqueles que apresentam evidência de invasão local macro ou microscópica no momento do diagnóstico inicial desenvolvem uma evolução clínica pior ${ }^{5}$.

\section{Tumores não seminomatosos}

Os TCGM não seminomatosos representam de $1 \%$ a 3,5\% de todos os tumores mediastinais e de $1 \%$ a 2\% de todos os TCG em homens².

Eles incluem: tumores de saco vitelino, carcinoma embrionário, coriocarcinomas e tumores de células germinativas não teratomatosos combinados (TCGC). Esses tumores são mais freqüentes em portadores da síndrome de Klinefelter e estão associados a um risco maior do desenvolvimento de neoplasias hematológicas, que não estão relacionadas ao tratamento quimioterápico sistêmico².

O tumor de saco vitelino primário do mediastino foi descrito inicialmente por Teilman et al. em 19676. Acomete, principalmente, homens jovens (idade de 15 a 35 anos), embora possa ocorrer em crianças e mulheres. Caracteriza-se clinicamente por massas mediastinais anteriores grandes e localmente invasivas. A sintomatologia dependerá do tamanho da lesão, sendo que os tumores maiores causam compressão de estruturas mediastinais adjacentes, enquanto que os menores podem ser assintomáticos ${ }^{2,7}$. 
Dentre as condições clínicas que podem estar associadas com os tumores de saco vitelino mediastinais, cita-se: síndrome de Klinefelter, precocidade do desenvolvimento sexual, distúrbios hematológicos (trombocitopenia idiopática) e neoplasias hematológicas ${ }^{7}$. Até 25\% dos pacientes podem apresentar metástases sistêmicas (fígado, pulmões e cérebro) ao diagnóstico. A AFP sérica encontra-se muito elevada em pacientes com essa condição, e o CEA pode ocasionalmente estar também elevado?.

Os carcinomas embrionários são os TCGM mais raros. Dificilmente apresentam-se em sua forma pura, sendo que na maioria das vezes estão associados com outros componentes de células germinativas. Afetam preferencialmente homens jovens (I5 a 35 anos) e podem apresentar características clínicas e radiológicas semelhantes aos tumores de saco vitelino, podendo ocorrer também em mulheres, pacientes mais idosos e crianças $^{2,7}$. São localizados preferencialmente no mediastino anterior, sendo geralmente muito grandes, circunscritos e invasivos.

Dentre os principais sinais e sintomas desses tumores, cita-se: hemoptises freqüentes, ginecomastia bilateral, atrofia testicular espermatogênica, precocidade do desenvolvimento sexual em crianças, acne extensa e síndrome de Klinefelter².

No que diz respeito aos marcadores tumorais, a AFP encontra-se mais freqüentemente elevada que a B-HCG.

Os carcinomas embrionários primários de mediastino apresentam um prognóstico ruim, com metástases pulmonares e linfáticas precoces ${ }^{2,3}$.

Os coriocarcinomas primários de mediastino são, em sua forma pura, os mais raros tumores de células germinativas do mediastino. As dificuldades em aceitar-se os coriocarcinomas como tumores primários mediastinais vieram da observação de que os primários testiculares podem se disseminar rapidamente antes de tornaram-se clinicamente detectáveis nas gônadas. Entretanto, é importante ressaltar que os tumores de células germinativas testiculares raramente se disseminam para 0 mediastino. A presença de uma grande massa mediastinal anterior sem achados no exame físico e ultrassonográfico dos testículos é altamente sugestiva de que a lesão seja primária do mediastino ${ }^{2,7}$.

Os coriocarcinomas primários de mediastino afetam homens jovens ( 15 a 35 anos de idade) e raramente mulheres. Geralmente não são muito grandes, a menos que estejam associados com outros elementos malignos de células germinativas. Tais tumores podem apresentar freqüentemente os seguintes sinais e sintomas: hemoptise, ginecomastia bilateral, atrofia espermatogênica dos testículos, precocidade do desenvolvimento sexual em crianças, acne extensa, além da possível associação com a síndrome de Klinefelter. A B-HCG sérica encontra-se muito elevada, e a AFP ocasionalmente elevada, principalmente quando tecidos glandulares imaturos estão presentes². Ao diagnóstico, a presença de metástases pulmonares bilaterais e extratorácicas é freqüente. São tumores com prognóstico muito ruim, sendo considerados os piores TCGM em termos de sobrevida².

Os tumores de células germinativas mediastinais combinados (TCGMC) não teratomatosos consistem na combinação dos vários tipos de tumores de células germinativas (incluindo os seminomas) na mesma lesão, sem a presença de elementos teratomatosos. Aparentemente, os TCGMC com componentes seminomatosos apresentam uma evolução menos agressiva.
O diagnóstico diferencial dos TCGM não seminomatosos/não teratomatosos citados anteriormente pode incluir uma série de condições clínicas.

Para o carcinoma embrionário, o mais importante diagnóstico diferencial é com a extensão local ou metástases de um carcinoma pouco diferenciado do pulmão. Dentre as características clínicas que podem ajudar nessa difereciação, incluem-se a idade do paciente (mais jovens para os pacientes com carcinoma embrionário) e a presença ou ausência de massa pulmonar, muitas vezes de difícil caracterização, já que muitos dos pacientes com carcinoma embrionário apresentam-se inicialmente com infiltração pulmonar importante ao diagnóstico. Estudos imunohistoquímicos podem ajudar nessa diferenciação?.

Os carcinomas de saco vitelino pouco comuns como os tipos hepatóide e intestinal, podem ser confundidos com metástases de carcinoma hepatocelular, carcinoma pouco diferenciado ou carcinoma papilar, respectivamente. História clínica minuciosa e estudos imunohistoquímicos ajudam a fazer um diagnóstico correto ${ }^{7}$.

Os coriocarcinomas são histopatologicamente distintos, e é importante diferenciá-los dos carcinomas pulmonares de grandes células com produção ectópica de HCG. A correlação clínica com a presença de massa pulmonar faz a diferenciação entre essas entidades?.

\section{Quadro clínico e diagnóstico}

Em uma análise internacional ${ }^{8}$ de 34 I pacientes com TCGM (de um total de 635 TCG extragonadais) seminomatosos e não seminomatosos, os sintomas mais comuns na apresentação inicial foram: dispnéia (25\%), dor torácica (23\%), tosse (17\%), febre (13\%), perda de peso ( $11 \%)$, síndrome de oclusão de veia cava (6\%), fraqueza ou cansaço (6\%) e dor em outras localizações além do tórax (5\%). Sintomas e sinais menos freqüentes foram: massa em parede torácica ou cervical (2\%), hemoptise, rouquidão, náuseas ou disfagia ( $1 \%$ cada). No que diz respeito aos exames radiológicos, a radiografia simples do tórax é quase sempre anormal na presença de um TCGM, sendo a vasta maioria dos tumores (ao redor de 95\%) localizados no mediastino anterior. A tomografia axial computadorizada de tórax com contraste endovenoso é o método de imagem de escolha na suspeita de um TCGM. A ressonância nuclear magnética geralmente não acrescenta mais informações relevantes do que as observadas no exame tomográfico?

Em relação aos exames laboratoriais, a dosagem dos marcadores séricos tumorais B-HCG e AFP é indispensável no diagnóstico e acompanhamento dos pacientes com TCGM? .

Os pacientes com teratoma benigno apresentam os marcadores negativos. Os seminomas mediastinais podem causar pouca elevação do B-HCG (geralmente menores que $100 \mathrm{UI} / \mathrm{ml})$. Qualquer elevação da AFP indica um componente tumoral não seminomatoso?.

As concentrações de AFP e B-HCG elevadas ou em elevação significam doença em atividade com a necessidade de tratamento subseqüente. Após quimioterapia ou cirurgia, as concentrações dos marcadores tumorais devem cair de acordo com suas meias-vidas: 5 a 7 dias para AFP e 30 horas para a B-HCG. Outro marcador tumoral presente em pacientes com TCGM é a desidrogenase lática $(\mathrm{DHL})$, que é menos específica que os demais citados acima?. 
Em geral, quando os marcadores tumorais estão elevados, o diagnóstico histológico é recomendado. Devido às dificuldades em diferenciar histopatologicamente os tumores de células germinativas dos carcinomas pouco diferenciados após a punção por agulha fina, a biópsia por agulha grossa (core biopsy) deve ser realizada sempre que possível. A mediastinotomia anterior, ou procedimento de Chamberlain, também pode ser realizada para acesso à lesão.

\section{Tratamento}

\section{Teratomas}

Os teratomas são tratados primeiramente por cirurgia, e a ressecção completa desses tumores proporciona um bom prognóstico, 91 .

A remoção cirúrgica dos teratomas maduros pode ser feita por toracotomia lateral ou esternotomia mediana, dependendo de tamanho, localização e extensão do tumor. Quando comprometem estruturas intratorácicas, como pulmão e pericárdio, podem ser de difícil remoção"!.

Os teratomas imaturos mediastinais são tumores potencialmente malignos e seu prognóstico é influenciado pela localização anatômica do tumor, a idade do paciente e a quantidade de componentes imaturos presentes no tumor. Quando ocorrem em pacientes com idade menor que 15 anos, apresentam comportamento biológico e clínico semelhantes aos teratomas maduros. Por outro lado, quando ocorrem em pacientes com idade maior que 15 anos, comportam-se como tumores altamente malignos. Para os pacientes portadores de teratomas imaturos, pode haver benefícios de quimioterapia baseada em cisplatina e radioterapia pós-operatória' ${ }^{12}$.

\section{Seminomas}

Os seminomas mediastinais são tumores sensíveis à radioterapia, tanto primária como adjuvante. Essa forma de tratamento tem se mostrado efetiva no controle desses tumores". Os seminomas primários de mediastino também apresentam boa resposta à quimioterapia com cisplatina como tratamento inicial, observando melhora na sobrevida tardia dos pacientes tratados quando comparados com o tratamento radioterápico primário ou adjuvante ${ }^{13,14}$. Existem vários tipos de tratamentos quimioterápicos propostos, bem como é variável a associação ou não desses esquemas com o tratamento radioterápico ${ }^{15}$. Diante da evidência de estadio avançado de doença, é normalmente indicado o tratamento quimioterápico primário".

Os seminomas mediastinais menores e encapsulados podem ser ressecados e submetidos à radioterapia adjuvante ${ }^{15}$.

Segundo Motzer et al. ${ }^{16}$, os pacientes com massas residuais acima de $3 \mathrm{~cm}$ devem ser operados, já que foi encontrado seminoma viável em $25 \%$ dos pacientes com essas características. O tratamento cirúrgico nesses casos preveniria a recorrência da doença e avaliaria os efeitos dos tratamentos de quimioterapia e radioterapia ${ }^{16}$.

Segundo a classificação do Consenso Internacional de Neoplasias de Células Germinativas ${ }^{17}$, a presença de metástases viscerais não pulmonares, principalmente para fígado e cérebro, indicam um prognóstico adverso para os tumores seminomatosos. As sobrevidas em cinco anos para os pacientes com metástases hepáticas e cerebrais estão ao redor de $54 \%$ e $57 \%$, respectivamente ${ }^{17}$. Para os pacientes sem metástases, a sobrevida em cinco anos é de aproximadamente $85 \%{ }^{17}$.

\section{Nãoseminomas}

A quimioterapia sistêmica com a combinação de bleomicina, etoposide e cisplatina (BEP) é considerada como o tratamento padrão para os TCGM não seminomatosos ${ }^{10}$. Foram observadas sobrevidas melhores em pacientes com resposta completa à quimioterapia baseada em cisplatina, e nos que apresentam declínio rápido dos marcadores tumorais ${ }^{10,11}$.

Devido ao fato de $30 \%$ dos pacientes com TCGM não seminomatosos não obterem uma resposta completa após as doses convencionais de cisplatina, Motzer et al. ${ }^{18}$, recomendam a utilização de altas doses de carboplatina e etoposide associadas ao transplante de medula óssea. Entretanto, essa forma de tratamento não foi bem sucedida em pacientes que apresentavam recorrência da doença pela segunda vez ou fossem refratários à cisplatina' ${ }^{19}$.

Segundo a classificação do Consenso Internacional dos Tumores de Células Germinativas ${ }^{17}$, os pacientes com tumores não seminomatosos de localização primária mediastinal representam uma entidade clínica distinta com fator prognóstico adverso (sobrevida em cinco anos ao redor de 40\%). Quando comparados com os outros pacientes com tumores de células germinativas (seminomas e não seminomas) de localização diferente, apresentam índices de resposta completa ao tratamento menores, bem como altos índices de recorrência e falência de terapia de resgate ${ }^{17}$.

Os pacientes com TCGM do tipo não seminomas que evoluírem com a normalização dos marcadores tumorais séricos após o tratamento quimioterápico e apresentarem massa persistente residual evidenciada na tomografia de tórax devem ser submetidos ao tratamento cirúrgico ${ }^{10}$.

Kesler et al. ${ }^{20}$ concluíram que o achado patológico pós-operatório de necrose completa da massa residual, bem como o de teratoma, correlacionavam-se com uma sobrevida excelente e boa, respectivamente. Essa sobrevida foi estatisticamente melhor do que a dos pacientes em que foram encontrados, no exame anatomopatológico pós-operatório, tumor de células germinativas persistente ou degeneração carcinomatosa/sarcomatosa.

Os teratomas residuais podem apresentar o potencial de crescer rapidamente e ocasionar complicações mecânicas na cavidade torácica conhecidas como growing teratoma syndrome ${ }^{21}$.

Os pacientes que apresentavam massa residual intratorácica e marcadores tumorais elevados eram tratados inicialmente com quimioterapia de resgate, mas não se observou melhora da sobrevida com essa abordagem ${ }^{10}$. Devido a tal fato, pacientes selecionados são considerados atualmente para o tratamento cirúrgico, mesmo na presença de marcadores tumorais elevados ${ }^{10,22}$. Em tais operações, são necessárias muitas vezes ressecções extensas de estruturas intratorácicas como o timo, pericárdio, pulmão, nervo frênico, veia inominada e cava superior ${ }^{10}$.

\section{Conflito de interesse: não há}




\section{SUMMARY}

\section{Primary mediastinal germ Cell tumors}

Primary mediastinal germ cell tumors accountforapproximately $10 \%$ to $20 \%$ of all neoplasms of the mediastinum. It is generally accepted that they represent the malignant transformation of germinal elements without a gonadal focus. These tumors can be classified as teratomas, seminomas, nonseminomatous (yolk sac tumor, embryonal carcinoma, choriocarcinoma) and combined germ-cell tumors without teratomatous components. This review purposes to discuss the different primary mediastinal germ-cell tumors, their clinical presentation and options for treatment. [Rev Assoc Med Bras 2006; 52(3): 182-6]

KEY wORDS: Mediastinal germ cell tumors. Mediastinum. Classification. Teratomas. Seminomas. Nonseminomatous.

\section{REFERÊNCIAS}

I. Moran CA, Suster S. Primary germ cell tumors of the mediastinum: I. Analysis of 322 cases with special emphasis on teratomatous lesions and a proposal for histopathologic classification and clinical staging. Cancer 1997:80:681-90.

2. Macchiarini $\mathrm{P}$, Ostertag $\mathrm{H}$. Uncommon primary mediastinal tumours. Lancet Oncol 2004;5: 107- 18.

3. Weidner N. Germ-cell tumors of the mediastinum. Semin Diagn Pathol 1999; 16:42-50.

4. Woolner LB, Jamplis RW, Kirklin JW. Seminoma (germinoma) apparently primary in the anterior mediastinum. N Engl J Med 1955; 252:653-7.

5. Moran CA, Suster S, Przygodzki RM, Koss MN. Primary germ cell tumors of the mediastinum: II. Mediastinal seminomas-a clinicopathologic and immunohistochemical study of I 20 cases. Cancer 1997;80:69 I-8.

6. Teilmann I, Kassis H, Pietra G. Primary germ cell tumor of the anterior mediastinum with features of endodermal sinus tumor. (Mesoblastoma vitellinum). Acta Pathol Microbiol Scand 1967;70:267-78.

7. Moran CA, Suster S, Koss MN. Primary germ cell tumors of the mediastinum: III. Yolk sac tumor, embryonal carcinoma, choriocarcinoma, and combined nonteratomatous germ cell tumors of the mediastinum-a clinicopathologic and immunohistochemical study of 64 cases. Cancer 1997;80:699-707.

8. Bokemeyer C, Nichols CR, Droz JP, Schmoll HJ, Horwich A, Gerl A, et al. Extragonadal germ cell tumors of the mediastinum and retroperitoneum: results from an international analysis. J Clin Oncol. 2002; 20: 1864-73.

9. Wright CD. Germ cell tumours of the mediastinum. In: Pearson FG, Cooper JD, Deslauriers J, Ginsberg RJ, Hiebert CA, Patterson GA, Vischel
HC, editors. Thoracic Surgery. 2nd ed. Philadelphia: Churchill Livingstone; 2002. p. I7। I-9.

10. Flores RJ. Surgical management of primary mediastinal germ cell tumours. General thoracic experts techiniques: CTSNET Experts' techniques; 2004. Avaliable from: http://www.ctsnet.org/doc/8875.2004.

I I. Takeda S, Miyoshi S, Ohta M, Minami M, Masaoka A, Matsuda H. Primary germ cell tumors in the mediastinum: a 50-year experience at a single Japanese institution. Cancer 2003; 97:367-76.

12. Dulmet EM, Macchiarini P, Suc B, Verley JM. Germ cell tumors of the mediastinum. A 30-year experience. Cancer 1993;72: | 894-90 I.

13. Gerl A, Clemm C, Lamerz R, Wilmanns W. Cisplatin-based chemotherapy of primary extragonadal germ cell tumors. A single institution experience. Cancer 1996;77:526-32.

14. Fizazi K, Culine S, Droz JP, Terrier-Lacombe MJ, Theodore C, Wibault P, et al. Initial management of primary mediastinal seminoma: radiotherapy or cisplatin-based chemotherapy? Eur J Cancer 1998;34:347-52.

15. Kiffer JD, Sandeman TF. Primary malignant mediastinal germ cell tumours: a literature review and a study of 18 cases. Australas Radiol 1999;43:58-68

16. Motzer R, Bosl G, Heelan R, FairW, Whitmore W, Sogani P, etal. Residual mass: an indication for further therapy in patients with advanced seminoma following systemic chemotherapy. J Clin Oncol 1987;5: I 064-70.

17. International Germ Cell Consensus Classification: a prognostic factorbased staging system for metastatic germ cell cancers. International Germ Cell Cancer Collaborative Group. J Clin Oncol 1997; I 5:594-603.

18. Motzer RJ, Mazumdar M, Gulati SC, Bajorin DF, Lyn P, Vlamis V, et al. Phase II trial of high-dose carboplatin and etoposide with autologous bone marrow transplantation in first-line therapy for patients with poor-risk germ cell tumors. J Natl Cancer Inst 1993;85: | 828-35.

19. Broun ER, Nichols CR, Einhorn LH, Tricot GJ. Salvage therapy with highdose chemotherapy and autologous bone marrow support in the treatment of primary nonseminomatous mediastinal germ cell tumors. Cancer 1991;68:1513-5.

20. Kesler KA, Rieger KM, Ganjoo KN, Sharma M, Fineberg NS, Einhorn LH, et al. Primary mediastinal nonseminomatous germ cell tumors: the influence of postchemotherapy pathology on long-term survival after surgery. J Thorac Cardiovasc Surg 1999; I | 8:692-700.

21. Afifi HY, Bosl G], Burt ME. Mediastinal growing teratoma syndrome. Ann Thorac Surg 1997;64:359-62.

22. Vuky J, Bains M, Bacik J, Higgins G, Bajorin DF, Mazumdar M, et al. Role of postchemotherapy adjunctive surgery in the management of patients with nonseminoma arising from the mediastinum. J Clin Oncol $2001 ; 19: 682-8$.

Artigo recebido: 17/02/2005

Aceito para publicação: 28/06/2005 